\section{Virtualizando la enseñanza presencial en Matemáticas. Recursos en bachillerato y nivel universitario}

\author{
Rocío Blanco Somolinos, José Luis González Fernández, Cristina Solares \\ Martínez
}

Universidad de Castilla La Mancha

\section{Resumen}

Se presentan dos recursos utilizados para transmitir los conocimientos a nuestros alumnos, una vez que se suspende la actividad docente presencial con motivo del coronavirus. El primer recurso, que se ha aplicado con un grupo de alumnos de Bachillerato, consiste en compartir las pantallas del ordenador y tablet, escribiendo con un lápiz óptico sobre esta última. El segundo recurso se ha aplicado a un grupo de alumnos de Universidad, empleando la metodología de aula invertida. Se graban vídeos explicando los conceptos y las clases, a través de la plataforma Teams, se dedican a repasar, resolver dudas y a realizar ejercicios. Los alumnos de ambos grupos han cumplimentado un cuestionario de satisfacción cuyos resultados confirman que su grado de satisfacción con las metodologías propuestas es muy alto. Les ha ayudado a aprender y dominar la materia, y las explicaciones les han parecido claras y ordenadas. Algunos consideran que se sienten nerviosos y que no es una diversión cursar una asignatura online. Además, no hay diferencias significativas entre ambos grupos, valorando los métodos de forma muy similar.

Palabras clave: Clase Virtual, Satisfacción estudiantil, Técnicas de aula, Aprendizaje online
Virtualizing classroom teaching in Mathematics. Resources in college and University levels

\section{Abstract}

This work presents two resources used to turn our screen into a whiteboard and thus transmit the knowledge to our students once the face-to-face teaching activity is suspended due to the coronavirus. The first resource, applied with a group of college students, consists of sharing the screens of the computer and Tablet, writing with a pencil on the latter as if it were a blank folio. The second resource, has been applied to a group of university students, using the methodology of flipped classroom, videos are recorded explaining the concepts and classes through platform Teams are devoted to review, resolve doubts, and perform exercises. Students from both groups have completed a satisfaction questionnaire whose results confirm that their degree of satisfaction with the proposed methodologies is very high. It has helped them to learn and master the subject, and teacher explanations have seemed them clear and orderly. Some students feel nervous and consider that it is not fun to take an online course. In addition, there are no significant differences between the responses of both groups, valuing both methods in a very similar way.

Key words: Virtual classrooms, Student satisfaction, Classroom Techniques, Online Learning

\section{Introducción}

El 12 de marzo de 2020, mediante el Decreto 8/2020, del Presidente de la Junta de Comunidades sobre medidas extraordinarias a adoptar con motivo del coronavirus (SARS-CoV-2), se acuerda la suspensión de la actividad docente presencial en todos los niveles educativos en Castilla-La Mancha desde el día 13 marzo y se ordena a la Consejería de Educación, Cultura y Deportes la adopción de las medidas oportunas para llevar a efecto este mandato.

Para ello, y con el fin de garantizar la continuidad del servicio educativo y minimizar el impacto para el alumnado matriculado, la Consejería de Educación, Cultura y

\title{
MAGISTER
}

Vol. 32. Núm. I: (2020). Sección extraordinaria 
Deportes de Castilla-La Mancha emite la Instrucción 1/2020 de 13 de marzo, donde dicta las siguientes instrucciones (p. 7237):

El periodo de suspensión temporal de la actividad escolar no debe entenderse como un periodo de interrupción de la actividad lectiva, por lo que hemos de procurar que el alumnado mantenga diariamente una rutina y unos hábitos de estudio en sus domicilios.

Con la finalidad de facilitar la continuidad del proceso de enseñanza y aprendizaje durante este período, la dirección de los centros educativos garantizará el diseño de planes de trabajo y material para que el alumnado pueda continuar trabajando de forma no presencial.

En Educación Secundaria, Bachillerato, FP, Adultos y Enseñanzas de Régimen Especial, el profesorado de cada materia coordinado con el profesorado tutor, preparará los materiales necesarios y establecerá contacto directo con el alumnado vía digital para hacer el seguimiento, trabajos de corrección, etc.

Por su parte, la Universidad de Castilla-La Mancha, emite el mismo 12 de marzo, una Resolución del Vicerrectorado de Docencia, donde "se proponen diversas alternativas con el fin de establecer un marco flexible que permita a los profesores adoptar las medidas que consideren más adecuadas para el desarrollo de las actividades de aprendizaje y evaluación, teniendo en cuenta las características específicas de sus asignaturas."

En dicha Resolución se recomienda el uso de las plataformas Campus Virtual y Microsoft Teams, y se indica que se habilitará una página web en el sitio web del Vicerrectorado de Docencia con recursos docentes y metodológicos procedentes de los Planes de Formación y del Centro de Tecnologías y Contenidos Digitales (C:TED) para facilitar la docencia a distancia.

En las líneas anteriores se ha hecho un resumen de todo lo que, los profesores, debíamos realizar el día 13 de marzo (viernes), para empezar/seguir nuestras clases no presenciales desde el lunes 16 de marzo de 2020 hasta la finalización del curso académico 2019/2020.

Como se puede observar, las indicaciones eran muy sencillas: "diseño de planes de trabajo y materiales para que el alumnado pueda seguir trabajando con normalidad". En cuanto al tiempo disponible para la preparación, siendo generosos, tres días, si contamos el sábado y el domingo (no lectivos).

¿Cuándo y cómo realizar la formación necesaria para esta nueva situación? Cuando se ha podido, mañanas, tardes, noches... y en el cómo, se ha vuelto a un aspecto importante: autoformación "sobre la marcha", es decir, según se ha ido desarrollando el proceso, se ha ido evolucionando en la forma de impartir las clases.

\section{Objetivos}

El objetivo fundamental para poder continuar con la docencia era convertir nuestras clases presenciales en clases virtuales en tiempo "récord". Para ello, se tuvo que responder a la pregunta ¿cómo dar clases de matemáticas online?

Una pregunta que, a priori, parecía sencilla, tan sencilla que, después de consultar numerosa bibliografía y webgrafía, llegamos a la conclusión de que había que disponer de:

1. Una buena conexión a internet (tanto alumnos como profesores). Cosa que no ha sido fácil.

2. Un aula virtual (disponíamos de plataformas como Moodle o el soporte de office 365).

3. Material elaborado, de forma expresa, para ser usado en estas circunstancias (en muchos momentos se tuvo que fabricar "sobre la marcha").

Sin embargo, aunque no lo parezca, estos factores no fueron los que dieron más problemas. El problema fundamental fue la forma de transmitir los conocimientos necesarios para que nuestros alumnos pudieran resolver todos los ejercicios, trabajos o tareas propuestas. ¿Qué hacer?

\section{MAGISTER}

Vol. 32. Núm. I: (2020). Sección extraordinaria 
“...el docente no es un mero transmisor de conocimientos, sino que además es un fuerte agente socializador y que, con su docencia transmite una serie de valores que van a calar, directa o indirectamente, en la formación de menores y jóvenes" (Prieto, 2008, p. 327).

Es precisamente en este período donde nos hemos dado cuenta y han cobrado una mayor importancia las palabras anteriores. Es aquí, donde los alumnos se han dado cuenta de la importancia de nuestra función, al margen de la transmisión de conocimientos, pero como el objetivo de este artículo es saber qué y cómo hemos hecho para transmitirlos, nos centraremos en esta última parte.

\section{Método}

Son numerosos los recursos y las formas que existen para las clases online, sin embargo y, dadas las circunstancias de confinamiento, cierre de instituciones, tiendas... fue complicado conseguir los materiales deseados para este fin. Es por eso por lo que, con lo que se disponía en casa (y alguna pequeña compra realizada por internet) nos dispusimos a dar nuestras clases, intentando imitar, en la medida de lo posible, las clases presenciales, puesto que es a lo que los alumnos estaban acostumbrados.

Nuestros compañeros de Historia y de muchas otras materias, usaban presentaciones en Power Point o elaboraban materiales que después subían a las plataformas que se mencionaron con anterioridad. Sin embargo, en el caso de Matemáticas, esto era complicado; los alumnos necesitaban la explicación del profesor, incluso muchos de ellos manifestaban la necesidad de ver esas explicaciones en la pizarra.

¿Cómo conectarnos con nuestros alumnos? Para conectar con nuestros alumnos se usó Microsoft Teams.

¿Cómo convertir nuestra pantalla en una pizarra para poder explicar? A continuación, presentamos dos propuestas para atajar esta cuestión, puestas en práctica a nivel de bachillerato y nivel universitario, aunque son adaptables a otros niveles educativos.

\section{MAGISTER}

Vol. 32. Núm. 1: (2020). Sección extraordinaria

\section{Pizarra virtual (con recursos caseros)}

En la asignatura de Matemáticas de $1^{\circ}$ de Bachillerato (Grupo 1) se utilizó un proceso que explicamos a continuación para convertir la pantalla de una Tablet en una pizarra.

1. Compartir la pantalla de la Tablet con el ordenador, para lo cual se utilizó la aplicación gratuita "Screen Mirror". Una vez descargada la aplicación en la Tablet, se abre (Figura 1).

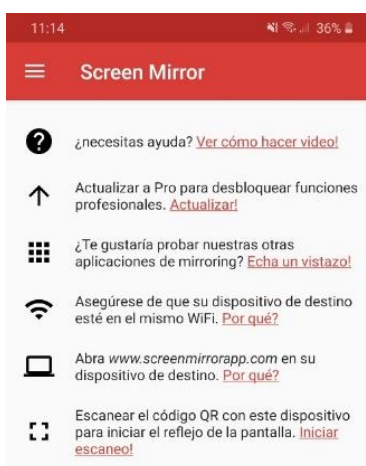

Figura 1. Pantalla inicial de Screen Mirror.

2. En el ordenador, desde cualquier navegador, accedemos a la web http://www.screenmirrorapp.com/. Una vez en la web, nos aparece una pantalla con un código QR que debemos escanear con la Tablet, utilizando la última opción de la aplicación abierta en la Tablet. 
Figura 2. Opción de escaneo.

3. Ya tenemos proyectada la pantalla de la Tablet (Figura 3) y, cualquier cosa que hagamos en la misma, se verá en el ordenador.

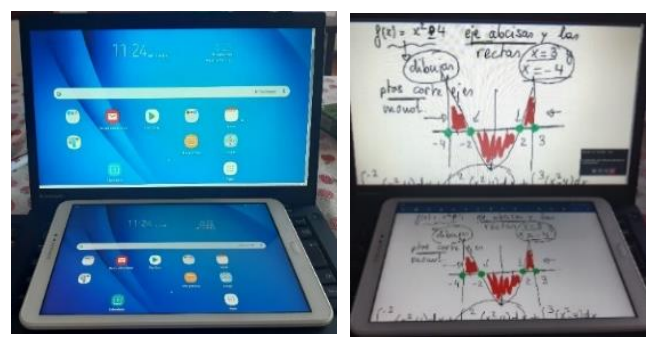

Figura 3. Pantalla compartida.

4. El siguiente paso nos llevaría a compartir pantalla con Microsoft Teams.

5. Una vez compartida la pantalla, viene otro de los pasos más importantes de este proceso, convertir nuestra pantalla de la Tablet en una pizarra lo más parecida a las que tenemos en clase. ¿Cómo hacerlo?

a) De nuevo, necesitamos instalar una aplicación en la Tablet (en nuestro caso, nos hemos decantado por Xodo) que nos permita escribir con el lápiz óptico.

b) Esta aplicación permite escribir sobre archivos con formato pdf, para lo cual, se creó un documento en blanco que nos sirviera de soporte en el que escribir. Por fin, todo listo para dar nuestra clase...

\section{Clase invertida virtual}

En la asignatura Instrumentos Matemáticos para la Ingeniería II de primer curso de un Grado de Ingeniería (Grupo 2), las clases se desarrollan combinando la plataforma Microsoft Teams con el uso de la plataforma Campus Virtual.

En Campus Virtual el profesor cuelga vídeos con la explicación de los conceptos que se repasarán en la clase. Además de vídeos con la resolución de ejercicios y vídeos en los que se muestra la aplicación de los conceptos teóricos. Se han realizado más de 100 vídeos cortos en los que se ve la mano del profesor que va explicando los conceptos escritos sobre un folio.

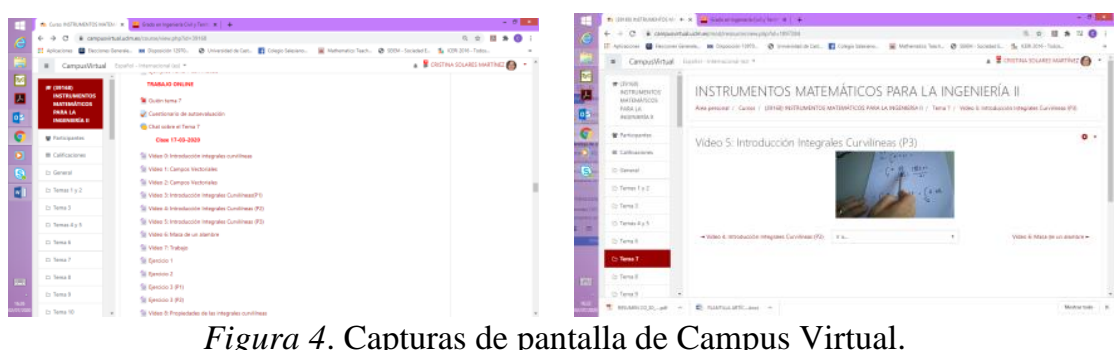

La metodología seguida en las clases combina la enseñanza tradicional explicando conceptos durante la clase online, con la metodología del aula invertida (Flipped Classroom) (Bergmann \& Sams, 2012; Wasserman et al., 2017), en la que los alumnos tienen disponibles todas las explicaciones grabadas en vídeo antes de las clases.

Como los alumnos pueden visualizar los vídeos antes de las clases, en las clases con Teams el profesor comparte un documento con los apuntes de cada tema y los ejercicios, explicando de forma breve los conceptos y los alumnos le plantean sus dudas. Además, durante las clases el profesor plantea ejercicios que los alumnos deben resolver en casa y los vídeos, con la resolución, se suben a Campus Virtual 
esa misma tarde, proporcionando un feedback constante al alumnado. Algunas dudas se resuelven con la pizarra de Teams, pero no nos parece un entorno adecuado para trabajar en Matemáticas.

En cada tema se incluye un cuestionario de Moodle, en el que se repasan los conceptos explicados en la clase virtual.

\section{Resultados}

Con esta forma de impartir clase se ha tratado de reproducir, lo más fielmente posible, un método que no resultase demasiado "diferente" a lo que los alumnos estaban acostumbrados. No se podía pasar de la "nada" al "todo" en dos días.

Los alumnos de los dos grupos han respondido a un cuestionario de satisfacción que se ha basado en el cuestionario de Auzmendi (1992). Tenían que puntuar las siguientes afirmaciones con valores entre 1 (Totalmente en desacuerdo) y 5 (Totalmente de acuerdo). Los resultados se recogen en las tablas 1 y 2.

Tabla 1.

Resultados del cuestionario, Grupo 1

\begin{tabular}{llllll}
\hline $\begin{array}{l}\text { Porcentajes de respuestas (Grupo 1, 33 } \\
\text { alumnos) }\end{array}$ & $\mathbf{1}$ & $\mathbf{2}$ & $\mathbf{3}$ & $\mathbf{4}$ & $\mathbf{5}$ \\
\hline $\begin{array}{l}\text { P1. La metodología utilizada por el profesor } \\
\text { motiva el trabajo continuo del alumno }\end{array}$ & 0 & 3 & 6,1 & 57,6 & 33,3 \\
\hline $\begin{array}{l}\text { P2. El profesor, con la metodología utilizada, } \\
\text { me ha ayudado a aprender }\end{array}$ & 0 & 0 & 0 & 33,3 & 66,7 \\
\hline $\begin{array}{l}\text { P3. Cursar una asignatura de forma online hace } \\
\text { que me sienta muy nervioso/nerviosa }\end{array}$ & 6,1 & 12,1 & 27,3 & 39,4 & 15,2 \\
$\begin{array}{l}\text { P4. Estudiar una asignatura en la modalidad } \\
\text { online es una diversión para mí }\end{array}$ & 30,3 & 33,3 & 36,4 & 0 & 0 \\
\hline $\begin{array}{l}\text { P5. Mi grado de satisfacción con la impartición } \\
\text { online de la asignatura es alto }\end{array}$ & 0 & 3 & 12,1 & 39,4 & 45,5 \\
\hline $\begin{array}{l}\text { P6. La metodología utilizada me ha ayudado a } \\
\text { dominar la materia }\end{array}$ & 0 & 3 & 18,2 & 51,5 & 27,3 \\
\hline $\begin{array}{l}\text { P7. Con las herramientas online utilizadas, el } \\
\text { profesor explica de forma clara y ordenada }\end{array}$ & 0 & 0 & 3 & 27,3 & 69,7 \\
\hline
\end{tabular}

P8. Si tuviera la oportunidad cursaría otras $6,1 \quad 12,1 \quad 15,2 \quad 27,3 \quad 39,4$ asignaturas impartidas con la metodología online utilizada por el profesor

P9. La metodología online se parece mucho a la docencia presencial impartida por el profesor P10. Si comparo la docencia presencial y online impartidas por el profesor, considero que asistiendo a las clases de forma presencial entiendo mejor las matemáticas

Tabla 2.

Resultados del cuestionario, Grupo 2

\begin{tabular}{lllllll}
\hline Porcentajes de respuestas (Grupo 2, 14 alumnos) & $\mathbf{1}$ & $\mathbf{2}$ & $\mathbf{3}$ & $\mathbf{4}$ & $\mathbf{5}$ \\
\hline P1
\end{tabular} $\begin{array}{lllll}\text { P1. La metodología utilizada por el profesor motiva el } & 0 & 0 & 14,3 & 42,9\end{array} \quad 42,9$ trabajo continuo del alumno

\begin{tabular}{llllll}
\hline P2. El profesor, con la metodología utilizada, me ha & 0 & 0 & 21,4 & 21,4 & 57,1
\end{tabular} ayudado a aprender

P3. Cursar una asignatura de forma online hace que $\begin{array}{llllll}0 & 7,1 & 21,4 & 71,4 & 0\end{array}$ me sienta muy nervioso/nerviosa

P4. Estudiar una asignatura en la modalidad online es $\quad \begin{array}{lllll}21, & 64, & 14,3 & 0 & 0\end{array}$ una diversión para mí

\begin{tabular}{lllll}
21, & 64, & 14,3 & 0 & 0 \\
4 & 3 & & & \\
\hline 0 & 7,1 & 28,6 & 50 & 14,3
\end{tabular}

P5. Mi grado de satisfacci

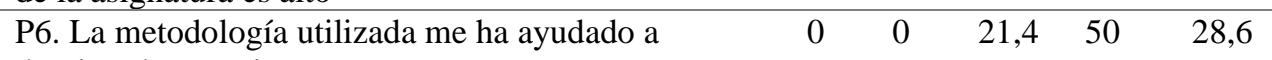

dominar la materia

$\begin{array}{llllll}\text { P7. Con las herramientas online utilizadas, el profesor } & 0 & 0 & 14,3 & 28,6 & 57,1\end{array}$ explica de forma clara y ordenada

$\begin{array}{llllll}\text { P8. Si tuviera la oportunidad cursaría otras } & 0 & 7,1 & 21,4 & 28,6 & 42,9\end{array}$ asignaturas impartidas con la metodología online utilizada por el profesor

$\begin{array}{llllll}\text { P9. La metodología online se parece mucho a la } & 0 & 21, & 14,3 & 28,6 & 35,7\end{array}$ docencia presencial impartida por el profesor P10.Si comparo la docencia presencial y online impartidas por el profesor, considero que asistiendo a las clases de forma presencial entiendo mejor las matemáticas 
Los resultados se han analizado con Statistical Package for Social Sciences, SPSS, v.25. Como podemos observar en la Figura 5, en ambos grupos se obtiene una distribución similar de las respuestas.
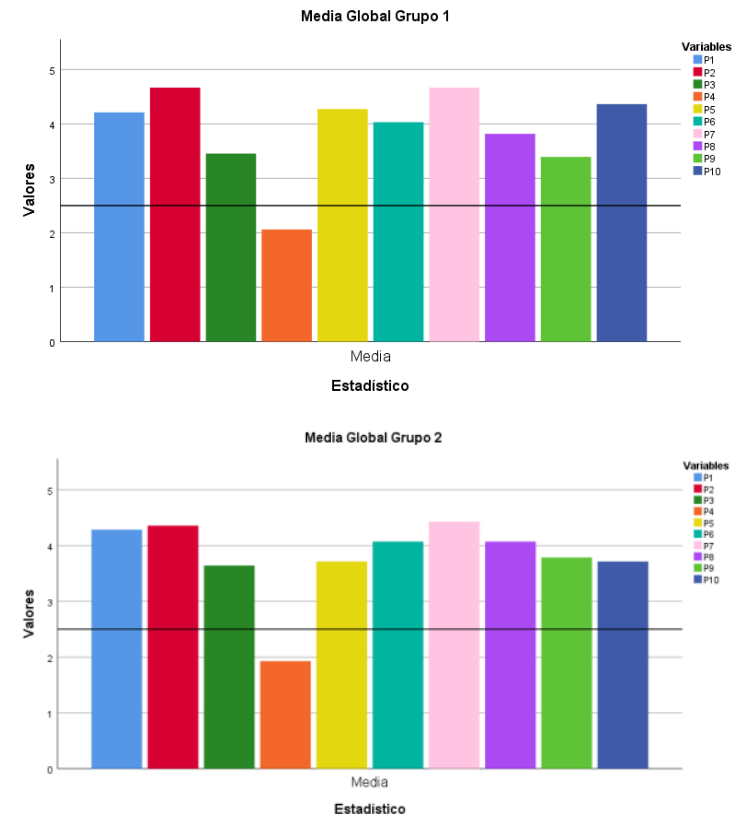

Figura 5. Medias globales en ambos grupos.

De hecho, la realización de pruebas no paramétricas muestra que no hay diferencias significativas entre las respuestas de ambos grupos en todas las cuestiones, salvo en la $\mathrm{P} 5(\mathrm{U}=144,000, \mathrm{p}=, 030)$ y la $\mathrm{P} 10(\mathrm{U}=151,000, \mathrm{p}=, 042)$ pero con un nivel de significación muy débil (Tabla 3 ).
Tabla 3.

Resultados del test de U Mann-Whitney

\begin{tabular}{lcccccccccc}
\multicolumn{1}{c}{} & P1 & P2 & P3 & P4 & P5 & P6 & P7 & P8 & P9 & P10 \\
\hline U de & 21 & 192, & 211, & 208, & 144, & 227, & 195, & 211, & 186, & 151, \\
Mann- & 8,0 & 500 & 500 & 500 & 000 & 500 & 000 & 500 & 000 & 000 \\
$\begin{array}{l}\text { Whitne } \\
\text { y }\end{array}$ & 00 & & & & & & & & & \\
\hline Sig. &, 736 &, 289 &, 626 &, 576 &, 030 &, 929 &, 314 &, 634 &, 279 &, 042
\end{tabular}

Si analizamos las respuestas de ambos grupos por separado, la prueba de Friedman muestra que hay diferencias significativas entre la distribución de las respuestas a lo largo de las 10 preguntas. Grupo $1\left(\chi_{9}^{2}=136,601, p=0,000\right)$, grupo $2\left(\chi_{9}^{2}=43,368\right.$, $\mathrm{p}=0,000$ )

Si nos fijamos en la figura 5, observamos que la puntuación de la pregunta P4 está por debajo de la media global y es muy inferior a las demás. En ambos grupos, las puntuaciones asignadas por los estudiantes a esta pregunta se distribuyen entre los valores 1 y 3 , el grado de acuerdo es bajo, para los alumnos no es divertido estudiar la asignatura de forma online.

Usamos la prueba de rangos con signo de Wilcoxon para comparar las preguntas. Se obtienen diferencias significativas entre el grado de ansiedad (P3) y agrado (P4), grupo $1(\mathrm{p}=0,000)$, grupo $2(\mathrm{p}=0,001)$. No así entre la motivación (P1) y la utilidad de la metodología $(\mathrm{P} 6)$, grupo $1(\mathrm{p}=0,180)$, grupo $2(\mathrm{p}=0,366)$ como era de esperar.

Si nos fijamos en las preguntas P9 y P10, más de un 50\% del alumnado les asigna puntuaciones entre 4 y 5 . En el grupo 1 la pregunta P9 obtiene un $51,5 \%$ y la pregunta $\mathrm{P} 10$ un $88 \%$; en el grupo 2 la pregunta P9 consigue un 64,3\% y la P10 un $50 \%$ de respuestas entre 4 y 5 . 


\section{Discusión}

Los resultados de los cuestionarios confirman que el grado de satisfacción de los estudiantes con las metodologías y herramientas utilizadas es muy alto.

Las puntuaciones medias de todas las preguntas, salvo la $\mathrm{P} 4$, se encuentran por encima de 3, y las puntuaciones medias de las preguntas P1, P2, P6 y P7 están por encima de 4 . Todo el alumnado ha puntuado estas cuestiones entre 3 y 5 , (salvo un $3 \%$ en el grupo 1 en las preguntas P1 y P6). Esto significa que consideran que la metodología empleada les ha motivado para trabajar, les ha ayudado a aprender y dominar la materia y las explicaciones del profesor les han parecido claras y ordenadas. Cabe destacar, que las puntuaciones medias de la pregunta P3 también están por encima de 3 , por lo que cursar la asignatura de forma online causa cierto grado de ansiedad en el alumnado.

Respecto a las preguntas P9 y P10, más de la mitad del alumnado considera que la metodología online empleada se parece mucho a la docencia presencial, pero prefieren la docencia presencial, siendo esta preferencia más acusada en el grupo 1 (bachillerato).

Esta diferencia pone de manifiesto la importancia del profesor en el proceso de enseñanza, cuya figura se percibe más relevante a nivel preuniversitario que a nivel universitario.

Si comparamos las puntuaciones de los cuestionarios de satisfacción para ambas opciones de enseñanza, no encontramos diferencias significativas, por lo que ambos métodos han sido valorados positivamente de forma similar por el alumnado.

\section{Conclusiones}

En este artículo presentamos dos formas de conseguir una "pizarra virtual" adecuada al trabajo en Matemáticas, para suplir la enseñanza presencial con explicaciones en la pizarra tradicional. A través de vídeos grabados con las explicaciones realizadas escribiendo sobre papel, o bien realizando dichas explicaciones en tiempo real escribiendo sobre una Tablet a modo de folio en blanco, se ha continuado con la docencia de forma virtual, de la manera más similar posible a la docencia presencial que se estaba llevando a cabo, intentando normalizar una situación que no era para nada normal. Los resultados obtenidos tras esta experiencia ponen de manifiesto la buena acogida a la metodología empleada por parte del alumnado participante.

En el escenario incierto en el que nos encontramos, esperamos que estas herramientas puedan ser de utilidad a otros docentes del área de Matemáticas.

\section{Referencias}

Auzmendi, E. (1992). Las actitudes hacia la matemática-estadística en las enseñanzas medias y universitarias. Bilbao: Mensajero, D.L.

Bergmann, J., \& Sams, A. (2012). Flip your classroom: Reach every student in every class every day. Alexandria, Egypt: ACSD and International Society for Technology in Education.

DOCLM (13/03/2020). Decreto 8/2020, de 12 de marzo, del Presidente de la Junta de Comunidades, sobre medidas extraordinarias a adoptar con motivo del coronavirus (SARS-CoV-2).

Instrucción 1/2020 de 13 de marzo, de la Consejería de Educación, Cultura y Deportes, para la aplicación de las medidas educativas por causa del brote del virus Covid-19 en los centros docentes de Castilla La Mancha.

Prieto, E. (2008). El papel del profesorado en la actualidad. Su función docente y social. Foro de Educación, 10, 325-345.

Resolución (12/03/2020) de la Vicerrectora de Docencia de la Universidad de Castilla - La Mancha, sobre las medidas extraordinarias previstas en coordinación con los Vicerrectorados competentes para adaptar la universidad a la suspensión temporal de la actividad educativa presencial.

Ruiz, P. (2010). El papel del maestro en el aula. Temas para la Educación. Recuperado de https://www.feandalucia.ccoo.es/docu/p5sd7499.pdf

Wasserman, N.H., Quint, C., Norris, S.A., \& Carr, T. (2017). Exploring flipped classroom instruction in Calculus III. International Journal of Science and Mathematics Educations, 15, 545-568.

*Autor de contacto: Rocío Blanco Somolinos, mariarocio.blanco@uclm.es

\section{MAGISTER}

Vol. 32. Núm. I: (2020). Sección extraordinaria 